\title{
Una aproximación a la cuantificación y caracterización del capital social: una variable relevante en el desarrollo de la provincia de Teruel, Espańa
}

\author{
María Isabel Saz-Gil. Universidad de Zaragoza, Teruel, España. \\ Juan David Gómez-Quintero. Universidad de Zaragoza, Zaragoza, España.
}

RESUMEN | El capital social tiene la capacidad efectiva de movilizar productivamente los recursos que radican en las distintas redes sociales a las que tienen acceso los miembros de una comunidad. Su presencia se relaciona con un amplio abanico de beneficios para individuos y sociedades; de la misma forma, su carencia puede implicar un freno al desarrollo. El objetivo de este trabajo es analizar y caracterizar el capital social en la provincia de Teruel (España), un territorio en el que predomina la vida rural y una economía altamente desarticulada. El método incluye la medición de los grados de confianza social existente y la estimación de la densidad asociativa, a través del análisis de fuentes secundarias. Los resultados ofrecen información sobre la solidez de las redes intracomunitarias y, simultáneamente, la precariedad de las redes extracomunitarias. Tales aspectos son necesarios de tener en cuenta en el diseńo de medidas que generen mayor capital social, un factor determinante para que la provincia consiga salir del círculo vicioso en el que se encuentra anclada.

PALABRAS CLAVE | redes, desarrollo territorial, desarrollo regional y local, capital social, confianza social, densidad asociativa.

ABSTRACT | Social capital has the capacity to mobilize the resources embedded in the social networks of the members of a community. The presence of this capital is related to a range of benefits to individuals and societies, while a lack of such capital may imply hampered develop $\neg m e n t$. The aim of this paper is to analyze and characterize the social capital of the province of Teruel (Spain), a rural territory characterized by a fragmented economy. The research methodology includes measuring the levels of social trust and estimating associational density through secondary sources analysis. The results provide information on the strength of intra-community networks and, simultaneously, the precariousness of extracommunity networks. These aspects must be considered when designing policies aimed at generating social capital, a cru-cial element for aiding the province in breaking the vicious cycle in which it is embroiled.

KEYWORDs | networks, territorial development, regional and local development, social capital, social trust, associational density. 


\section{Introducción}

En general, se acepta la tesis de que en todo proceso de desarrollo es necesario utilizar de forma imaginativa, racional, equilibrada y dinámica todas las formas de capital y bienes patrimoniales, ya sean estos monetarios, humanos, naturales, culturales, sociales o territoriales (Comisión Económica para América Latina y el Caribe [CEPAL], 1992). El concepto de capital social cobra fuerza en esta perspectiva, puesto que en una economía global, donde la mayoría de los recursos tienen gran movilidad, aquellos que están arraigados en el territorio, como el capital social o el medioambiental, constituyen la principal ventaja competitiva de las zonas en que ejercen su influencia (Bryden, 1998; Malecki, 2011).

Camagni (2008) conceptualiza la idea de "capital territorial", cuya función económica es reforzar la eficiencia y la productividad de las actividades locales, y hacerlo de un modo diferenciado según los distintos espacios de que se trate. El autor divide las capacidades territoriales en diferentes familias de capitales y, con el fin de proporcionar una clasificación exhaustiva de todas las fuentes potenciales de lo que podría ser "el capital territorial", establece una matriz de tres por tres, construida sobre dos dimensiones principales: de un lado, el nivel de materialidad de los bienes; y del otro, el grado de rivalidad entre ellos. En esa clasificación se encuentra el capital social al lado de los bienes intangibles y con carácter de bien colectivo. Tal como indica Camagni (2008), existe una gran herencia teórica en torno al desarrollo endógeno y a sus factores específicos e intangibles, que muchas veces han sido reinterpretados en forma de capital social (Putnam, 1993). Con estos planteamientos se han desarrollado investigaciones sobre distritos industriales (Sforzi, 2002), sistemas productivos locales (Garofoli, 1992) y medios innovadores (Maillat, 1995).

El capital social permite a las regiones iniciar una espiral dinámica de desarrollo (Sweeney, 2001). Desde esta perspectiva, se relaciona con un amplio abanico de beneficios y posibilidades para individuos y sociedades en aspectos como el logro educativo y ocupacional, la salud física y mental, la reducción de las tasas de delincuencia, el acceso al mercado o la movilidad y logro laboral, etcétera (Coleman, 1988; 1990; Fukuyama, 1995; Putnam, 1993; 1995). Puede ser entendido, así, como las características de la vida social -redes sociales, normas y confianza- que permiten a los participantes en ella actuar asociados de una forma más efectiva para conseguir objetivos compartidos (Putnam, 1993). Como indican Woolcock y Narayan (2000), si el individuo entra en contacto con sus vecinos, y estos con nuevos vecinos, se producirá una acumulación de capital social que puede satisfacer sus necesidades sociales y producir nuevas posibilidades para mejorar las condiciones de vida de toda la comunidad. Así considerado, el capital social viene a convertirse en un bien público que se debe promocionar, mientras la carencia del mismo puede implicar un freno al desarrollo.

La densidad de las relaciones entre diversos agentes económicos y sociales basadas en la confianza mutua y la capacidad de cooperación es lo que contribuye a establecer las ventajas comparativas de que goza un área rural concreta. La red de relaciones sociales existentes en un contexto social configura la manera en que el aprendizaje colectivo y la innovación tienen lugar (Falk \& Kilpatrick, 2000; 
Westlund, 2006). El trabajo de Putnam (1993) puso el capital social en el centro de la discusión académica al explorar el rol de la sociedad civil en la determinación de los desequilibrios en el desarrollo regional. En este sentido, Camagni (2003) analiza los múltiples canales a través de los cuales las diferentes categorías de capital social pueden tener influencia en el desarrollo local. De hecho, se puede utilizar el concepto de capital social con un fin instrumental, como parte de políticas que persiguen la puesta en marcha de proyectos de desarrollo y contra la pobreza, según lo han hecho organismos como el Banco Mundial. También se puede analizar su formación a través de la intervención pública mediante la promoción y creación de redes de participación ciudadana (Skocpol, 1997).

El objetivo de este trabajo es analizar y caracterizar el capital social en la provincia de Teruel en España. Este estudio pretende arrojar luz sobre las formas de relación social, de articulación de los recursos sociales y de los niveles de confianza en virtud de los cuales la región pueda fortalecer su espiral de desarrollo. El documento se ha organizado de la siguiente forma. Tras la Introducción, el apartado segundo se ocupa de ilustrar el marco conceptual y profundizar en la confianza y en el ámbito asociativo como indicadores del capital social. En el apartado tercero se plantean los rasgos caracterizadores de la provincia de Teruel. El cuarto apartado presenta la metodología seguida. Los apartados quinto y sexto se ocupan respectivamente de analizar la confianza social y las organizaciones asociativas en la provincia de Teruel. Por último, se resumen los principales hallazgos y conclusiones.

\section{Marco conceptual del capital social}

El estudio del capital social ha dado lugar a dos corrientes que entienden el concepto de modo distinto, según su respectiva visión de las dimensiones o categorías de fenómenos que le atribuyen (Ostrom, 1999; Uphoff, 2000). La corriente estructuralista, que se sitúa fundamentalmente en el marco de la sociología y la economía - principalmente los trabajos de Bourdieu (1985) y Coleman (1990)-, lo define como un conjunto de recursos disponibles para el individuo, derivados de su participación en redes sociales. La otra corriente, desarrollada por los estudios realizados desde la ciencia política (Torcal \& Montero, 2000), es denominada enfoque culturalista. Ello obedece a que se centra en las normas, reglas y valores sociales (básicamente confianza, reciprocidad y civismo) compartidos por los miembros de una comunidad. Son aspectos de carácter más subjetivo que los considerados por la aproximación estructuralista, y que en general facilitan las relaciones.

Bourdieu (1980) señala que el capital social es el conjunto de los recursos actuales o potenciales vinculados a la posesión de una red duradera de relaciones más o menos institucionalizadas de interconocimiento e interreconocimiento; o, dicho de otro modo, vinculados a la pertenencia a un grupo en tanto en cuanto conjunto de agentes que no solo poseen propiedades comunes, sino que también están unidos por lazos permanentes y útiles. Coleman (1988) destaca tres aspectos relevantes del capital social: (i) el contexto de obligaciones, expectativas y confianza de los actores; (ii) la calidad de la información a la que acceden; y (iii) la disponibilidad de normas y sanciones efectivas para marcar las relaciones. Su trabajo, sin embargo, subraya en 
particular el carácter eminentemente relacional del capital social. Para este autor, un individuo posee un capital si, y solo si, el interlocutor reconoce y acepta el valor de cambio de ese capital; en este caso tiene que darse un elemento mínimo de reconocimiento y de relación válida; por tanto, de significados socialmente compartidos. Abundando en esta idea, Sobel (2002) reitera que el capital social hace referencia a esas condiciones y circunstancias en las cuales los individuos pueden recurrir a su pertenencia a grupos y redes para asegurarse beneficios. De esta forma, el capital social tiene un carácter relacional, pero una utilidad particular. Se trata de algo construido y creado de manera circunstancial por la integración en grupos, organizaciones y redes, que permite a los sujetos obtener rendimientos personales.

La distinción de Putnam (2000) entre capital social de tipo bonding (de unión) y capital social de tipo bridging (puente) se ha convertido en un referente en los estudios sobre el capital social (Growiec \& Growiec, 2014).

El capital social de tipo bonding remite a los vínculos sociales entre colectivos cercanos, similares y familiares. Se identifica habitualmente con redes densas de carácter multifuncional. La reciprocidad que en él se da es a largo plazo. Por lo que respecta a la confianza, se trata de confianza particularizada; se constatan fuertes normas compartidas por el grupo y la relación social entre los miembros del grupo no tiene carácter meramente instrumental (Leonard \& Onyx, 2003).

Por su parte, el capital social de tipo bridging se refiere a vínculos sociales establecidos entre grupos disímiles, alejados y no familiares. Muestra diferencias sensibles respecto del anterior: en él la densidad de la red de relaciones es baja, la reciprocidad se manifiesta en el corto plazo, la confianza es de carácter generalizada, las normas compartidas se sustentan en el conocimiento, y la relación social entre los agentes tiene un carácter instrumental (Leonard \& Onyx, 2003).

Woolcock (2001) amplía la distinción de Putnam (2000) e introduce el capital social de tipo linking (de vinculación o conexión), que constituye la capacidad para apalancar recursos, ideas e informaciones desde las instituciones formales con diferentes posiciones de jerarquía o autoridad.

Coleman (1988) señala que el capital social propio de las estructuras sociales cerradas tiene ventajas para los miembros del grupo; sin embargo, resulta poco probable que se genere a partir de estas estructuras una cultura de confianza generalizada, ya que el capital social de tipo bonding tiene carácter excluyente. Cuando los grupos están centrados en sí mismos sin relaciones con otros, se incrementa la presencia de capital social de tipo bonding. Granovetter (1973; 1985) insiste sobre la mayor importancia de las redes débiles frente a las redes fuertes y estructuras cerradas, puesto que las primeras permiten el acceso a información y conocimiento más novedosos que las últimas. Las redes débiles son especialmente importantes para unir miembros de diferentes grupos pequeños, mientras que los vínculos fuertes tienden a concentrarse en grupos particulares. En la misma línea, Burt (2005) afirma que el capital social tipo bridging se relaciona más positivamente que el tipo de capital bonding con el rendimiento económico de los individuos, la creatividad, la confianza social y la felicidad.

De otro lado, varios autores (Durston, 1999; 2000; Woolcock, 1998) estiman que hay diferentes formas de capital social, basándose para ello en la distinción 
entre lo individual y lo colectivo. Dasgupta (1999) habla del capital social grupal u organizacional, entendido como bien privado, pero con significativas externalidades positivas, y del capital social comunitario, entendido como bien público que beneficia a toda la sociedad. En este sentido, Durston (1999) argumenta que el capital social es un fenómeno comunitario, debido a que las instituciones locales de cooperación y cogestión surgen como un resultado frecuente de la interacción de estrategias individuales. Sin embargo, como indica Buciega (2010), la generación de capital social y su transformación en acción colectiva dirigida al desarrollo no es algo fácil de conseguir. Triglia (2001) señala que un determinado contexto territorial puede resultar más o menos rico en capital social, en función del grado en que los individuos y colectivos que allí residen se implican en redes relacionales más o menos difundidas. A través de las redes se puede aumentar el sentido de confianza y responsabilidad entre los actores con presencia activa en los distintos ámbitos, constituyéndose así la base para una acción colectiva que puede influir decisivamente en los procesos de desarrollo. Portes (2004) sostiene que el capital social de una comunidad tiene dos manifestaciones principales: la solidaridad confinada y la confianza exigible. La primera es el nivel de lealtad desplegada por los miembros entre sí, que produce apoyo mutuo en las relaciones con el mundo externo. La segunda es la seguridad de que se cumplirá con las obligaciones individuales, debido al poder sancionador de la comunidad. La amenaza de aislamiento es una garantía que suple la formalidad contractual.

De acuerdo con Herreros y De Francisco (2001), la generación de capital social puede darse mediante la intervención de un agente externo - por ejemplo, el gobierno local (Warner, 2001) - y como subproducto de la realización de otras actividades así, fortalecer las instituciones es una forma de inversión en capital social (Ostrom, 1994; 1999)-. La formación del capital social como subproducto de la realización de otro tipo de actividades, que no supongan la superación previa de un dilema de la acción social, es la solución habitual de los autores fundacionales del concepto (Coleman, 1988, 1990; Putnam, 1993), de forma que lo plantean como un resultado no intencional de la acción intencional. Coleman (1988) señala como un indicio de la consolidación de una red de capital social la producción de un resultado lateral de la asociación inicial, de forma que la asociación primaria es apropiada para usos alternativos no previstos en su origen. Asimismo, las organizaciones no lucrativas pueden desempeñar un papel clave, facilitando el establecimiento de nuevas formas de colaboración entre la comunidad y los organismos gubernamentales (Warner, 2001).

La tarea de medir el capital social resulta compleja, puesto que al no ser un elemento tangible, se carece de elementos identificativos claros que correspondan a parámetros de medida definidos. A este respecto, Camagni (2003) explora la problemática del análisis cuantitativo del capital social, indicando que la acepción relacional resulta más susceptible de ser medida, mientras que los aspectos de carácter macro o sistémicos son útiles como conceptos de externalidades genéricas. En el momento de proceder a su cuantificación se utilizan variables aproximativas, al igual que se hace con el capital humano, pero en el caso del capital social todavía 
no existe unanimidad sobre cuál o cuáles son las variables más adecuadas ${ }^{1}$. En sus trabajos acerca del declive del capital social en Estados Unidos, Putnam (1995; 2000) se inclina por los indicadores de densidad asociativa y la confianza social, también empleados por otros estudios afines (Hall, 1999; Torcal \& Montero, 2000).

\section{La confianza como indicador del capital social}

La confianza generalizada -esto es, la creencia en la benevolencia de los seres humanos en general (Yamagishi \& Yamagishi, 1994)- es un importante predictor del desarrollo socioeconómico (Fukuyama, 1995; Putnam, 1993). Facilita las transacciones económicas entre extrańos, ya que permite realizar una evaluación positiva de la fiabilidad en el cumplimiento de los compromisos formales adoptados (Zucker, 1986). Implica no solo ausencia de motivos oportunistas, sino también la creencia en que la otra parte se preocupa por el bienestar de la primera. Se reconoce que ese tipo de expectativas influye en el comportamiento de los individuos, de forma que cuando una parte percibe que la otra se preocupa por su bienestar, esta percepción no solo proporciona la base a partir de la cual un individuo decide confiar en otro (Gambetta, 1988), sino que además el individuo que confía desarrolla una especial sensibilidad hacia las necesidades personales y profesionales de aquellos individuos que considera dignos de su confianza, además de un deseo de responder a esas necesidades de forma tangible (Shaw, 1997).

Algunos trabajos establecen la existencia de correlación entre confianza generalizada y confianza en instancias políticas (Baquero, 2003; Montero, Calvo \& Martínez, 2008). La confianza política supone una evaluación positiva de los atributos más relevantes que hacen a cada institución digna de confianza, como credibilidad, justicia, competencia y transparencia (Montero et al., 2008). La confianza política en una institución conlleva la creencia de que esta no actuará de una forma arbitraria o discriminatoria que resulte dañina para los intereses propios o los de la región, sino que tratará a los ciudadanos de forma igualitaria y justa (Montero et al., 2008). La teoría que relaciona confianza social y confianza en la política sostiene que las instituciones funcionan mejor en una cultura con predisposiciones positivas a la cooperación recíproca. Además, afirma que la desconfianza entre los representantes elegidos y la comunidad afecta seriamente la estructuración de una sociedad (Baquero, 2003). Las definiciones de confianza existentes muestran que las creencias que llevan a una parte a confiar en otra son múltiples, por lo que se la considera un constructo multidimensional, siendo difícil encontrar consenso entre los investigadores respecto a las dimensiones que la configuran (Lewicky, Mcallister \& Bies, 1998).

\section{El nivel de asociacionismo como indicador del capital social}

Algunos estudios empíricos que relacionan la pertenencia a asociaciones voluntarias con la confianza generalizada (Brehm \& Rahn, 1997; Stolle \& Rochon, 1998) parecen confirmar la hipótesis de Putnam, según la cual las asociaciones más

1 La técnica más generalizada mediante la que se mide el capital social es a través de encuestas. Entre las iniciativas emprendidas se encuentra el Cuestionario Integrado para la Medición del Capital Social, del Banco Mundial, en 2002; y el Estudio de Referencia del Capital Social Comunitario (The Social Capital Community Benchmark, SCCB), de la Escuela Pública de la Kennedy School. 
heterogéneas generan más confianza social. Por otra parte, no se confirma la idea de que fuertes relaciones de confianza dentro del grupo sean la base de las expectativas de confianza social. Tal como había concluido Granovetter (1973) en su análisis de las redes, los vínculos fuertes, caracterizados por altos niveles de confianza, pueden ser un obstáculo para el desarrollo de vínculos más débiles, siendo estos los que pueden poner más recursos en manos del individuo.

Otros estudios establecen una vinculación efectiva entre densidad asociativa y capital social (Hooghe, 2003; Sobel, 2002). Saz Gil (2007) distingue diversas tipologías asociativas con diferente potencial para generar capital social; Van Deth (2006) muestra que el efecto del capital social sobre el grado de satisfacción en la democracia, sobre el interés por la política y la centralidad de la política en la vida de los individuos, depende del tipo de asociación en la que se participa. De esta forma, algunas asociaciones son especialmente relevantes para ampliar la densidad de las redes sociales, los participantes en otras desarrollan valores especialmente significativos para el apoyo de las instituciones democráticas, mientras que otras organizaciones entrenan a los individuos en determinadas habilidades cívicas.

\section{Área de estudio}

La Comunidad Autónoma de Aragón está integrada por las provincias de Huesca, Zaragoza y Teruel. Esta última está situada en la zona nororiental de la península ibérica, limita con la provincia de Zaragoza y las comunidades autónomas de Cataluña, Comunidad Valenciana y Castilla-La Mancha. Su capital es la ciudad de Teruel. Geográficamente la provincia se encuentra en el área meridional del Sistema Ibérico, aunque una pequeña porción del noreste pertenece a la Depresión del Ebro. La provincia de Teruel está dividida en diez comarcas (Sierra de Albarracín, AndorraSierra de Arcos, Bajo Aragón, Bajo Martín, Cuencas Mineras, Gúdar-Javalambre, Jiloca, Maestrazgo, Matarraña, Comunidad de Teruel) (Figura 1).

En el año 1900, la provincia tenía una población de 251.994 habitantes; en 2011, de 144.607 (Instituto Nacional de Estadística [INE], 2011). La historia de Teruel está caracterizada por la escasez y dispersión de la población. Hace algunos años, un grupo de investigadores afirmaba que algunas comarcas de Teruel estaban sumidas en un círculo vicioso caracterizado por el despoblamiento, el envejecimiento, la merma de actividad o la falta de iniciativa, que conducían a la escasez de oportunidades para sus habitantes, en especial para jóvenes y mujeres (Pereira, Fernández, Ocón \& Márquez, 2004).

En buena parte de estas comarcas, el proceso de pérdida de población ha comportado la disminución de la población social y económicamente activa (Gómez-Quintero \& Sanz-Gracia, 2013). Entre las debilidades y condicionantes de desarrollo de la provincia de Teruel, se encuentra la deficiente dotación y mantenimiento de infraestructuras viarias y de comunicaciones y la despoblación continuada, que genera escasez de recursos humanos, bajo dinamismo demográfico y creciente envejecimiento de la población, todo lo cual implica encarecimiento en el mantenimiento de servicios básicos, sociales, sanitarios y culturales en múltiples municipios de la provincia (Querol, 2006). 
FIgURA I Localización de la provincia de Teruel y comarcas que la integran

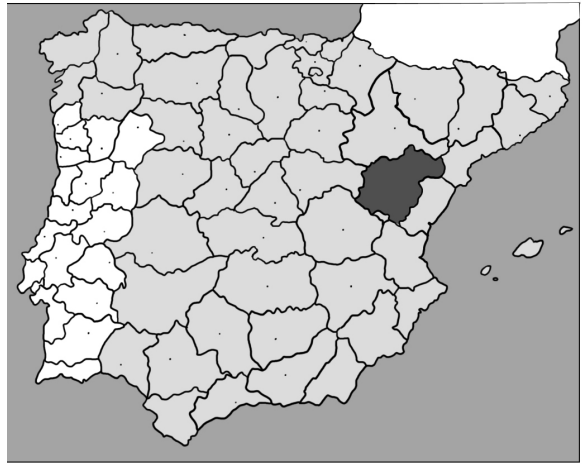

FUENTE HTTP://WWW.LUVENTICUS.ORG/MAPAS/ PENINSULAIBERICA/TERUEL.HTML;

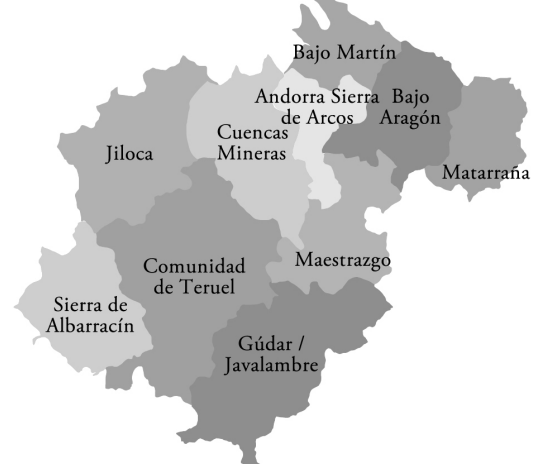

FUENTE HTTP://WWW.ZONU.COM/ DETAIL/2OIO-IO-O8-I 22 I 6/ COMARCAS-DE-LA-PROVINCIA-DETERUEL.HTML

Aragón tiene la consideración de unidad territorial de nivel 2 según la nomenclatura de unidades territoriales estadísticas (NUTS 2), de acuerdo con la definición establecida en el Reglamento (CE) 1059/2003. La Unión Europea reconoce diferentes tipos de regiones específicas que se enfrentan a problemas de desarrollo debido a que presentan desventajas relativas por sus condicionantes geográficos, debilidades estructurales, problemas de accesibilidad y alejamiento de los centros demográficos y de servicios importantes (Burillo, Burillo \& Ruiz, 2013). Estas regiones tienen características geográficas fácilmente identificables ${ }^{2}$. Como indica el Programa de Desarrollo Rural de Aragón 2014-2020, conforme al nuevo criterio de ruralidad establecido por la Comisión Europea en 2010, mediante el cual clasifica el territorio a nivel de NUTS 3 (nivel provincial) en tres grupos -regiones predominantemente urbanas, regiones intermedias y regiones predominantemente rurales-, la provincia de Teruel se considera predominantemente rural, porque su población rural representa más del $50 \%$ de la población total provincial.

No obstante, de acuerdo con las investigaciones realizadas por Burillo et al. (2013) en el marco del proyecto "Serranía Celtibérica" ${ }^{3}$ la mayor parte de la provincia de Teruel presenta las condiciones de región montañosa, rural remota y despoblada (por debajo de 8 habitantes por kilómetro cuadrado), con una situación de envejecimiento que la posiciona como un territorio desestructurado de la Unión Europea. De ser incorporada tal caracterización en la definición de políticas públicas, la provincia de Teruel podría recibir mayor número de ayudas económicas para abordar la situación; no obstante, el proyecto mencionado no ha conseguido el respaldo oficial suficiente y no ha sido considerado por el Gobierno de España

2 Las características de las regiones se pueden consultar en Monfort (2009), disponible en http:// goo.gl/5uSqUd

3 Serranía Celtibérica se desarrolla a partir del Proyecto I+D+i Segeda y Serranía Celtibérica, del Instituto Celtiberia de Investigación y Desarrollo Rural. El Instituto tiene entre sus objetivos, la promoción de este territorio (http://www.celtiberica.es/). 
como una Inversión Territorial Integrada (ITI) (Burillo, 2014). Esta situación ha sido reiterada, ya que la provincia de Teruel ha dispuesto, a largo plazo, de menos fondos para inversiones en infraestructuras que otros territorios, se le ha exigido un mayor esfuerzo para cofinanciar las actuación en las que participan los Fondos Europeos, y ha contado con menores subvenciones públicas para incentivar la inversión empresarial (Querol, 2006) ${ }^{4}$.

No obstante lo anterior, la provincia de Teruel se ha beneficiado de forma importante de políticas comunitarias de desarrollo en el medio rural, como la iniciativa comunitaria LEADER (Lazos Entre Acciones del Desarrollo de la Economía Rural), que tiene por objeto atender las zonas rurales más desfavorecidas a través de un enfoque innovador, contando para ello con la participación y corresponsabilidad de los agentes sociales en el proceso de programación de sus propias estrategias territoriales de desarrollo. En cuanto a las aportaciones de la iniciativa LEADER en la provincia de Teruel, además de la económica, Querol (2006) indica que se han realizado contribuciones significativas en los procesos de concienciación social, de participación ciudadana en estrategias de desarrollo endógeno a escala comarcal, así como en la mejora de la autoestima de la población rural.

Para la gestión de la iniciativa LEADER se crearon expresamente los Grupos de Acción Local (GAL), asociaciones basadas en los principios de participación y representatividad, e integradas en la Red Aragonesa de Desarrollo Local ${ }^{5}$. Desde nuestro punto de vista, la contribución de LEADER a la generación de capital social ha sido significativa, ya que ha fomentado el asociacionismo empresarial, turístico, agroalimentario y cultural en las comarcas.

\section{Metodología}

En el trabajo se ha utilizado gran variedad de fuentes secundarias, en particular datos procedentes de las siguientes fuentes: Barómetro del Centro de Investigaciones Sociológicas (CIs) de septiembre de 2010, n 2844; índice de confianza del consumidor de 2011, realizado por el Instituto de Crédito Oficial; Barómetro de Opinión de Aragón realizado en la primavera de 2011 (A+M, 2011); estadísticas electorales del Ministerio del Interior, a partir de cuyos registros el Instituto Aragonés de Estadística (IAEST, 2011) elaboró los datos de las votaciones; Encuesta sobre Discapacidades y Autonomía personal (INE, 2008); estadísticas del sector no lucrativo en Aragón (IAest, 2000); bases de datos del Gobierno de Aragón de asociaciones, fundaciones y colegios profesionales, además de trabajos previos del grupo de investigación GeSEs (Grupo de Estudios Sociales y Económicos del Tercer Sector) de la Universidad de Zaragoza. A partir de los datos recabados se ha realizado un análisis descriptivo.

4 Aragón y, por consiguiente, la provincia de Teruel quedó fuera de los Fondos Estructurales Europeos a través del Objetivo 1, quedando enmarcada en el objetivo 5 b.

5 La Red (RADR) surgió en 1995, siendo la primera Comunidad de España que impulsó la creación de una asociación en torno a los Grupos de Desarrollo Rural. Su finalidad es favorecer los intercambios de experiencias y alentar la cooperación para el desarrollo de las zonas rurales. 


\section{Análisis de la confianza en la provincia de Teruel}

El Barómetro del cis recoge en algunas oleadas variables como los niveles de confianza de los españoles hacia algunas personas, organizaciones y servicios, así como el número medio de personas con las que cuentan los españoles en caso de necesidad económica, sanitaria, afectiva o familiar. En concreto, el barómetro de septiembre de 2010, en el estudio número 2844, destaca que las personas en las que más confían los españoles son sus familiares, seguidos por los amigos, los compañeros de trabajo y los vecinos. En un segundo nivel aparecen las organizaciones y asociaciones, especialmente las organizaciones solidarias y de voluntariado (Cuadro 1).

CUADro I | Nivel de confianza de los españoles en caso de necesitar ayuda (de 0 , ninguna confianza, a 10 , total confianza)

\begin{tabular}{|c|c|}
\hline & MEDIA \\
\hline Familiares & 8,70 \\
\hline Amigos/as & 7,60 \\
\hline Compañeros/as de trabajo y/o estudios & 6,18 \\
\hline Vecinos/as & 5,88 \\
\hline Organizaciones de ayuda o voluntariado (Cruz Roja, etc.) & 5,73 \\
\hline Servicios sociales públicos & 5,32 \\
\hline Organizaciones religiosas (parroquia, Cáritas, etc.) & 4,42 \\
\hline
\end{tabular}

A pesar de que el estudio 2844 del cis no habla concretamente de las comarcas aragonesas, sí discrimina algunas variables territoriales, como la diferencia entre el entorno rural y el urbano. En tal sentido, podemos afirmar que en las zonas rurales españolas, como es el caso de la provincia de Teruel, las personas tienen mayores niveles de confianza. Para estudiar los niveles de confianza en la provincia de Teruel nos hemos basado en tres indicadores, que proceden de estudios diferentes. El primero de ellos es el nivel de confianza en el presente y en el futuro económico de los turolenses $(A+M, 2011)$. En segundo lugar, el nivel de confianza en los representantes políticos a partir del comportamiento electoral (IAEST, 2011, con datos del Ministerio del Interior). Y, por último, el nivel de confianza en las redes sociales (familiares, vecinos y amigos) ante situaciones de enfermedad (INE, 2008).

\section{Confianza en el presente y en el futuro económico}

Las percepciones individuales son una fuente de información valiosa para estimar las perspectivas de crecimiento económico, tal y como sugieren los índices de confianza del consumidor ${ }^{6}$. Una de las cuestiones que se aprecia con claridad de uno de los Barómetros de Opinión de Aragón (A+M, 2011), es que los turolenses son más pesimistas frente al futuro económico que el resto de los habitantes de la

6 Periódicamente, el Instituto de Crédito Oficial de España mide este índice de percepción subjetiva. http://www.ico.es/web/contenidos/0/160/index.html [en línea]. Consulta 16 de octubre de 2011. 
Comunidad Autónoma de Aragón. Los turolenses perciben de forma más acusada el pesimismo económico a nivel personal que el resto de los aragoneses. Mientras que el $74,8 \%$ de estos últimos cree que el futuro económico seguirá igual o un poco peor $^{7}$, el $79 \%$ de los turolenses tiene esta misma percepción. Respecto a los ciudadanos que perciben un claro empeoramiento de la situación económica personal, las diferencias son menos evidentes. La percepción del empeoramiento (poco y mucho) se da en el 20,9\% de los turolenses y en el 20,2\% de los aragoneses (Cuadro 2).

CUADRo 2 $\mid$ Confianza en el futuro de la situación económica personal en Teruel y Aragón (porcentajes)

\begin{tabular}{|l|c|c|}
\cline { 2 - 3 } \multicolumn{1}{c|}{} & TERUEL & ARAGón \\
\hline Mejorará mucho & 0,7 & 1,2 \\
\hline Mejorará un poco & 14,4 & 15,9 \\
\hline Se mantendrá igual & 61,1 & 58,4 \\
\hline Empeorará un poco & 17,7 & 16,4 \\
\hline Empeorará mucho & 3,2 & 3,8 \\
\hline NS/NC & 2,9 & 4,3 \\
\hline Total & 100,0 & 100,0 \\
\hline
\end{tabular}

FUENTE ELABORACIÓN PROPIA A PARTIR DE BARÓMETRO DE OPINIÓN DE ARAGÓN (A+M). PRIMAVERA 2OII. N = TERUEL 589 , ARAGÓN 2529

Al margen de la situación personal, la percepción del futuro económico de la comunidad autónoma es algo paradójica. El segmento de las personas optimistas disminuye cuando se les pregunta por la futura situación económica de Aragón. El bloque de los que consideran que la situación seguirá igual, aumenta, y el bloque de los pesimistas disminuye. El dato que se mantiene constante es el pesimismo de los turolenses frente al conjunto de los aragoneses. Mientras que el 83,9\% de los aragoneses cree que su comunidad estará igual o peor en el próximo año, esa proporción se incrementa hasta el 87,8\% en el caso de la opinión de los turolenses (Cuadro 3).

CUADro 3 | Confianza en el futuro de la situación económica de Aragón en comparación con el resto de Espańa. Teruel y Aragón (porcentajes)

\begin{tabular}{|l|c|c|}
\cline { 2 - 3 } \multicolumn{1}{c|}{} & TERUEL & ARAGóN \\
\hline Mejor & 8,7 & 10,8 \\
\hline Peor & 15,6 & 15,0 \\
\hline Igual & 72,2 & 68,9 \\
\hline NS/NC & 3,6 & 5,3 \\
\hline Total & 100,0 & 100,0 \\
\hline
\end{tabular}

FUENTE ELABORACiÓN PROPIA A PARTIR DE BARÓMETRO DE OPINIÓN DE ARAGÓN (A+M). PRIMAVERA 2OI I. N = TERUEL 589 , ARAGÓN 2529 


\section{Confianza en los representantes políticos}

Ante la dificultad para obtener datos comarcales que relacionen directamente la confianza social en los representantes políticos, se utiliza un indicador indirecto; esto es, la participación electoral como síntoma de confianza y la abstención electoral como síntoma de desconfianza. A partir de la teoría revisada sobre el capital social y confianza política o institucional (Baquero, 2003; Montero et al., 2008; Van Deth, 2006), es factible formular esta interrelación.

Los resultados del abstencionismo en las elecciones municipales y autonómicas de 2011 muestran que el tamaño de la unidad de población está relacionado con el grado de confianza política. Aquellas comarcas turolenses con menor población (Maestrazgo, Sierra de Albarracín...) tienen niveles de abstención inferiores al 20\%, mientras que las comarcas más pobladas (Comunidad de Teruel y Bajo Aragón) tienen niveles de abstención superiores al 25\% (Cuadro 4). En el caso de toda la comunidad autónoma de Aragón, la abstención fue del 30,56\%; en la provincia de Zaragoza, del 31,83\%; y en el conjunto del Estado, del 33,77\%. Estos datos supondrían una mayor confianza política en las unidades territoriales pequeńas y cierta desconfianza en las unidades territoriales más grandes. Cuando los ciudadanos conocen personalmente a sus candidatos tienden a incrementar su confianza a través del voto, ya que el contacto personal que no está mediatizado solo por la propaganda electoral (prensa, radio, TV e internet) tiende a reforzar los lazos sociales. Como seńalan Montero et al. (2008), la confianza social es el elemento central en un complejo círculo en el cual la reciprocidad y la confianza se asocian con la participación social y la implicación en asuntos cívicos. Estas conductas contribuyen a construir las instituciones sociales y políticas necesarias para unos gobiernos democráticos y eficientes; y estos, a su vez, crean las condiciones en las cuales puede florecer la confianza social y política.

CUADro 4 Población de las comarcas turolenses que se abstuvo en las elecciones municipales 2011 (porcentajes), y población total

\begin{tabular}{|l|c|c|}
\hline \multicolumn{1}{|c|}{ COMARCA } & ABSTENCIONES (\%) & POBLACIÓN TOTAL (N) \\
\hline Bajo Martín & 21,0 & 7.135 \\
\hline Jiloca & 23,3 & 14.142 \\
\hline Cuencas Mineras & 21,9 & 9.094 \\
\hline Andorra-Sierra de Arcos & 24,3 & 11.523 \\
\hline Bajo Aragón & 25,3 & 30.311 \\
\hline Comunidad de Teruel & 30,3 & 46.957 \\
\hline Maestrazgo & 19,5 & 3.682 \\
\hline Sierra de Albarracín & 14,7 & 4.930 \\
\hline Gúdar-Javalambre & 16,1 & 8.610 \\
\hline Matarraña/Matarranya & 21,9 & 8.893 \\
\hline
\end{tabular}

FUENTE ELABORACIÓN PROPIA A PARTIR DE MINISTERIO DEL INTERIOR; IAEST, 20 I I 
En un informe sobre las comarcas de Aragón (López, Ansó, Minguijón, Nocetti \& Ulldemolíns, 2006) se señaló que la cercanía de los ámbitos de decisión política a las comarcas redundaría en mayores posibilidades de agrandar el campo donde impera la democracia, es decir, mayor poder de decisión y mayor dotación de recursos. El Cuadro 4 muestra que la hipótesis planteada se confirma en la mayoría de las comarcas analizadas (salvo en Cuencas Mineras y Maestrazgo). Es decir, cuanto mayor es el tamaño de la población, mayor es la abstención y, por tanto, mayor la desconfianza.

\section{Redes sociales y atención a la enfermedad}

En los trabajos sobre capital social, la familia figura como principal núcleo de apoyo en todas las sociedades (Marcuello, Marcuello, Bellostas \& Moneva, 2007), tendencia a la cual no escapa España (CIs, 2010). Las redes sociales personales están integradas principalmente por familiares, amigos, vecinos y compañeros de trabajo. Estas redes son clave en situaciones de necesidad, como enfermedad, obtención de un préstamo de dinero o búsqueda de empleo ${ }^{8}$. Sin embargo, dentro de las redes personales, la familia destaca como el elemento clave, constituye un núcleo de apoyo fundamental, es la institución y grupo en que más se confía y al que se recurriría en caso de necesidad. Por ello se tiene en cuenta la Encuesta sobre Discapacidades, Autonomía personal y situaciones de Dependencia (INE, 2008), que ofrece datos a nivel provincial y pregunta por las redes sociales de los discapacitados. Uno de los primeros resultados que llama la atención es que de las tres provincias aragonesas, Teruel aparece como aquella en que son los familiares, vecinos y amigos los que con mayor frecuencia realizan visitas personales a quienes padecen una discapacidad (Cuadro 5).

CUADro 5 | Población aragonesa con discapacidad según la frecuencia con la que ha visto a alguno de sus familiares/amigos/vecinos

\begin{tabular}{|l|r|r|r|r|r|r|r|r|}
\cline { 2 - 9 } \multicolumn{1}{c|}{} & \multicolumn{2}{c|}{ ARAGóN } & \multicolumn{2}{c|}{ HUESCA } & \multicolumn{2}{c|}{ TERUEL } & \multicolumn{2}{c|}{ ZARAGOZA } \\
\cline { 2 - 10 } \multicolumn{1}{c|}{} & \multicolumn{1}{c|}{$\mathrm{N}$} & \multicolumn{1}{c|}{$\%$} & \multicolumn{1}{c|}{$\mathrm{N}$} & \multicolumn{1}{c|}{$\%$} & $\mathrm{~N}$ & $\%$ & $\mathrm{~N}$ & $\%$ \\
\hline $\begin{array}{l}\text { Todos los días o casi todos } \\
\text { los días }\end{array}$ & 91.354 & 81,9 & 12.047 & 77,2 & 8.128 & 86,8 & 71.180 & 82,1 \\
\hline 1 o 2 veces por semana & 12.423 & 11,1 & 1.079 & 6,9 & 509 & 5,4 & 10.835 & 12,5 \\
\hline 1 o 2 veces por mes & 2.518 & 2,3 & 152 & 1,0 & 180 & 1,9 & 2.186 & 2,5 \\
\hline Con menor frecuencia & 801 & 0,7 & 57 & 0,4 & 43 & 0,5 & 702 & 0,8 \\
\hline $\begin{array}{l}\text { No tiene familiares/ } \\
\text { amigos/vecinos }\end{array}$ & 3.235 & 2,9 & 2.191 & 14,0 & 443 & 4,7 & 601 & 0,7 \\
\hline No consta & 1.249 & 1,1 & 73 & 0,5 & 66 & 0,7 & 1.110 & 1,3 \\
\hline $\begin{array}{l}\text { Total población con } \\
\text { discapacidad }\end{array}$ & 111.581 & 100,0 & 15.599 & 100,0 & 9.368 & 100,0 & 86.614 & 100,0 \\
\hline
\end{tabular}

FUENTE ELABORACIÓN PROPIA A PARTIR DE ENCUESTA SOBRE DISCAPACIDADES, AUTONOMÍA PERSONAL Y SITUACIONES DE DEPENDENCIA (INE, 2008). UNIDADES: PERSONAS DE 6 Y MÁS AÑOS grantes en EE.UU. Sus estudios indican que el espacio familiar crea condiciones que hacen factible una estrategia clave de supervivencia entre los inmigrantes a través del autoempleo. La familia minimiza los costos de producción, transacción e información asociados con el mismo, y facilita la aparición de empresas operadas familiarmente. 
Por tanto, considerando la dimensión relacional del capital social (Camagni, 2003) y la dimensión comunitaria (Durston, 1999), podríamos señalar que la provincia de Teruel dispone de un capital social más elevado que las otras provincias, ya que destacan la frecuencia e intensidad de las relaciones personales a nivel familiar, fraternal y vecinal en el cuidado de personas que necesitan atención. La provincia aragonesa no solo es aquella donde es más frecuente la visita de quienes componen las redes sociales, sino la que brinda mejores posibilidades de relación con familiares y personas próximas. Buena parte de las posibilidades de relación cercana en la población turolense tiene que ver con el arraigo rural de las personas mayores de ochenta ańos, que son las que suelen presentar las tasas más altas de discapacidad. La elevada frecuencia de visita entre los miembros que pertenecen a las redes sociales de los discapacitados y la también elevada posibilidad de relaciones entre familiares y personas cercanas contrasta con el débil intercambio de relaciones hacia las personas desconocidas. Esta tendencia puede traducirse en que las redes sociales de apoyo son fuertes hacia el interior de los grupos sociales y núcleos territoriales, pero que estos grupos se encuentran más desconectados de redes puente que aquellos de las otras provincias aragonesas; esto es, desconectados de aquellos sistemas que los vincularían con estructuras de relación hacia el exterior. Al respecto podemos hablar de redes intracomunitarias fuertes y redes extracomunitarias débiles. Por otro lado, hay que decir que, según la Encuesta sobre Discapacidades citada, Teruel es la provincia aragonesa en la que más discapacitados permanecen en el domicilio familiar, en una proporción que llega al 55\%. Esto se puede explicar atendiendo a la ausencia de disponibilidad de recursos especializados en el medio rural (residencias y centros de día), aunque también al elevado arraigo familiar existente.

Después de haber observado los niveles de confianza económica, política e interpersonal, haremos una aproximación a la cuantificación del capital social desde la dimensión asociativa, esto es, la estimación de los niveles de agregación voluntaria como expresión de la confianza generalizada en el territorio seleccionado.

\section{Análisis del nivel de asociacionismo}

Para el análisis del tejido asociativo de la provincia, se distingue entre organizaciones de primer y de segundo nivel. Desde un punto de vista jurídico-formal, las organizaciones objeto de estudio pueden constituirse de manera diversa. Por lo general, suelen hacerlo a través del derecho de asociación recogido en el artículo 22 de la Constitución Espańola y regulado con carácter general a través de la Ley 1/2002, de 22 de marzo (Boletín Oficial del Estado [вов] de 26 de marzo). Junto al derecho de asociación, otro de los cauces para la constitución jurídica de las organizaciones en referencia es el derecho de fundación que reconoce el artículo 34 de la Constitución у que regula la Ley 50/2002, de 26 de diciembre (воЕ de 27 de diciembre).

\section{Análisis de las organizaciones de primer nivel}

Como señalan López et al. (2006), en algunas comarcas se ha detectado, como fruto del proceso de comarcalización de Aragón, el surgimiento de fórmulas de participación compleja, a través de consejos participativos o consultivos (en Turismo, en 
Deportes, en Juventud, etcétera), a imagen de los que ya vienen desarrollándose en otras administraciones, considerando incluso instancias de planificación estratégica con amplia participación de la ciudadanía. El proceso de comarcalización señalado ha estimulado el crecimiento de este tipo de organizaciones en las zonas rurales de Teruel. Aunque su origen se remonta al siglo XIX, su generalización se produjo en España a partir de la democracia y su consolidación a finales del siglo xx y principios del siglo XXI. Las organizaciones de la sociedad civil, también denominadas como organizaciones del tercer sector, u organizaciones sociales, son un fenómeno más urbano que rural y tienen más auge en las ciudades medianas y grandes que en los municipios pequeños.

Para medir la importancia de las organizaciones asociativas se utiliza la tasa de asociacionismo, entendida como el número de organizaciones constituidas formalmente por cada 1.000 habitantes. La ventaja que reporta esta tasa es que permite ver el número de entidades registradas sin que se diluyan los datos absolutos sobre el total de la población. El Cuadro 6 muestra que la media de asociaciones de la provincia es de 9,2 entidades por cada 1.000 habitantes. Las comarcas más destacadas son Gúdar-Javalambre y Sierra de Albarracín, con 16,7 y 15,4 entidades por cada 1.000 habitantes, respectivamente. Es muy significativo que las dos comarcas con las tasas de asociacionismo más altas sean, simultáneamente, las mismas dos que registran las tasas de abstención electoral más bajas de la provincia (IAEST, 2011, con datos del Ministerio del Interior).

CUADro 6 | Tasa de asociacionismo en las comarcas de Teruel (número de asociaciones/1.000 habitantes)

\begin{tabular}{|l|c|}
\hline \multicolumn{1}{|c|}{ COMARCA } & TASA DE ASOCIACIONISMO \\
\hline Bajo Martín & 14,3 \\
\hline Jiloca & 9,9 \\
\hline Cuencas Mineras & 9,6 \\
\hline Andorra-Sierra de Arcos & 10,8 \\
\hline Bajo Aragón & 5,4 \\
\hline Comunidad de Teruel & 7,9 \\
\hline Maestrazgo & 11,1 \\
\hline Sierra de Albarracín & 15,4 \\
\hline Gúdar-Javalambre & 16,7 \\
\hline Matarrańa/Matarranya & 10,0 \\
\hline PROVINCIA TERUEL & 9,2 \\
\hline
\end{tabular}

FUENTE ELABORACIÓN PROPIA A PARTIR DE ESTADÍSTICAS DEL SECTOR NO LUCRATIVO EN ARAGÓN (IAEST, 2000)

Así, como puede apreciarse en el Gráfico 1, la comarca Comunidad de Teruel es, históricamente, la que reporta un mayor número de organizaciones, y es en la que se sitúa Teruel, la capital de la provincia. La siguen las comarcas que ostentan una mayor población, por lo que, efectivamente, se constata la tendencia a una presencia 
creciente de estas entidades en aquellas con más población. En el conjunto de la provincia, el incremento de las organizaciones de la sociedad civil ha sido espectacular, y no ha parado de aumentar en los últimos treinta ańos.

\section{GRÁfICO I Distribución de organizaciones sociales por fecha de creación en las comarcas de Teruel}

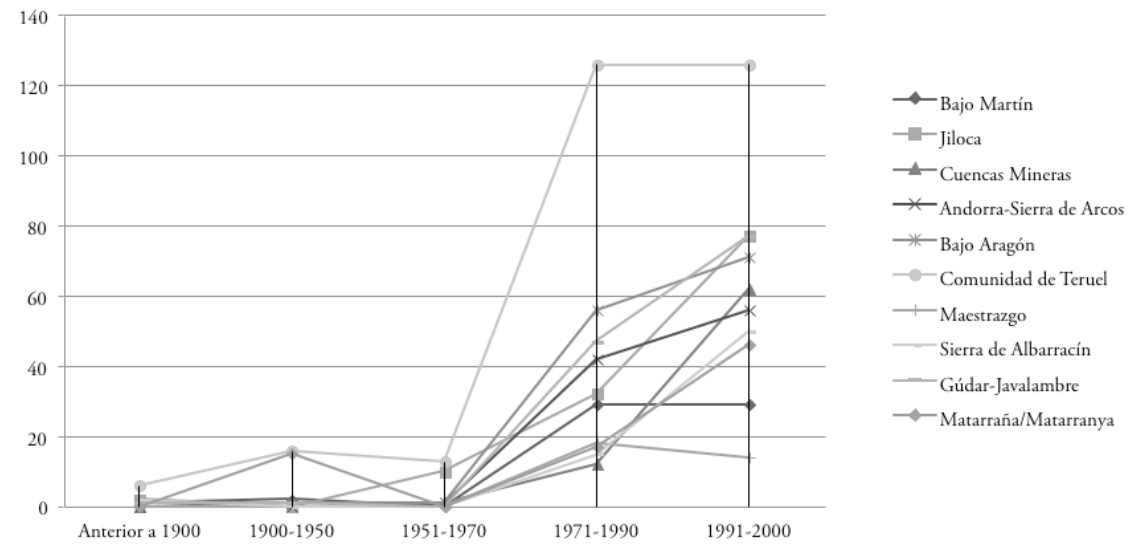

FUENTE ELABORACIÓN PROPIA A PARTIR DE ESTADÍSTICAS DEL SECTOR NO LUCRATIVO EN ARAGÓN (IAEST, 2000)

Por lo que respecta a los sectores de actividad en los que se integran las organizaciones sociales, una parte de ellas tiene carácter multisectorial, es decir, están dirigidas a un amplio abanico de población. Otra gran parte está constituida por entidades especializadas en actividades particulares (cultura, deporte, padres y madres de alumnos, servicios sociales, vecinos, mujeres mayores, jóvenes, discapacitados e inmigrantes).

En cuanto a la tipología de las organizaciones de la sociedad civil, la segunda forma jurídica más habitual después de las asociaciones, es la de fundaciones. Estas son entes de carácter corporativo o, como expresamente define la Ley, "organizaciones constituidas sin fin de lucro que, por voluntad de sus creadores, tienen afectado de modo duradero su patrimonio a la realización de fines de interés general" (art. 1, Ley 50/2002). En el caso de Aragón, la provincia de Teruel tiene registradas en el directorio de Fundaciones de Aragón unas cuarenta entidades cuyos intereses van desde la paleontología, pasando por la promoción del cine de Luis Buñuel, hasta la celebración de las Bodas de Isabel. Con todo, solo representan el 11\% de las fundaciones aragonesas.

\section{Organizaciones de segundo nivel: federaciones y plataformas}

Las organizaciones de segundo nivel existentes en la provincia de Teruel ponen en evidencia el nivel de maduración del asociacionismo. El Cuadro 7 muestra la situación de este tipo de entidades, donde destaca particularmente la desproporción del tamaño de este nivel entre la provincia de Zaragoza y las dos provincias 
restantes. Zaragoza acoge al 72,5\% de las federaciones y plataformas de Aragón, Huesca el $14,7 \%$ y Teruel tan solo el 12,8\%.

Otro elemento que llama la atención en el Cuadro 7 es la alta proporción de organizaciones en situación de inactividad. Ello se debe a que muchas veces la constitución de una organización es relativamente sencilla, pero su continuidad suele implicar un esfuerzo económico y personal arduo y cotidiano, que sus fundadores no logran soportar. Así, si bien no desaparecen, permanecen inactivas. La provincia de Teruel es la única en la que las federaciones y plataformas inactivas superan a las activas 9 .

CUADRO 7 | Distribución geográfica de las federaciones/plataformas en Aragón, 2010

\begin{tabular}{|l|c|c|c|c|c|c|}
\cline { 2 - 7 } \multicolumn{1}{c|}{} & ACTIVAS & $\%$ & INACTIVAS & $\%$ & SUBTOTALES & $\%$ \\
\hline Zaragoza & 54 & 49,54 & 25 & 22,93 & 79 & 72,47 \\
\hline Huesca & 9 & 8,25 & 7 & 6,422 & 16 & 14,67 \\
\hline Teruel & 4 & 3,66 & 10 & 9,17 & 14 & 12,84 \\
\hline Total & $\mathbf{6 7}$ & $\mathbf{6 1 , 4 6 7}$ & 42 & 38,53 & 109 & 100 \\
\hline
\end{tabular}

FUENTE ELABORACiÓN PROPIA A PARTIR DE MARCUELlo (2OIO), CON DATOS DEL REGISTRO DE ASOCIACIONES EN ARAGÓN

\section{Discusión y conclusión}

El capital social es un recurso intangible arraigado en el territorio y con carácter de bien colectivo. Por ello viene a convertirse en una variable relevante en el desarrollo de cualquier territorio cuya presencia conviene promocionar, y la carencia del mismo puede implicar un freno al desarrollo. En este trabajo nos hemos aproximado a la cuantificación y caracterización del capital social en la provincia de Teruel. A pesar de la escasez de fuentes de información que permitan medir los niveles de capital social en territorios específicos, es posible realizar aproximaciones a ello. En este trabajo se ha realizado tal tarea en dos dimensiones del capital social que tienen una estrecha relación teórica. Por una parte, se han conjugado mediciones de tres tipos de confianza por parte de los habitantes del territorio seleccionado: confianza en el futuro económico, en las instituciones políticas (a partir de la participación electoral), y en las redes personales de atención y cuidado. De otro lado, se ha analizado otra manifestación del capital social en su dimensión grupal u organizacional a través del grado de asociacionismo por sectores y niveles de la provincia. Estas medidas de confianza y de asociacionismo retratan el perfil de un territorio que requiere mejorar sus niveles de desarrollo económico.

El perfil construido a lo largo del estudio evidencia una moderada confianza por parte de la población hacia un mejor futuro del empleo y de la situación económica

9 Es significativo mencionar la plataforma "Teruel Existe", un movimiento reivindicativo que se ha hecho escuchar en toda España para exigir un mayor compromiso inversor de las Administraciones Públicas con la provincia. 
del territorio. Hay que señalar que las expectativas de recibir un tratamiento financiero más favorable por parte de los Fondos Europeos para mejorar sus opciones de desarrollo se han visto frustradas de forma continuada (Querol, 2006; Burillo et al., 2013), por lo que el horizonte futuro de la provincia no resulta muy halagüeño. Por el contrario, se ha apreciado que las redes personales de atención y cuidado -familiares, vecinos y amigos- son fuertes hacia el interior de los grupos sociales y núcleos territoriales, pero se encuentran más desconectadas que en otras provincias respecto de otras redes externas de relación grupal y organizacional (asociaciones, fundaciones y federaciones).

El estudio muestra la existencia de redes intracomunitarias fuertes a la par que redes extracomunitarias débiles. Es decir, se constata la existencia en la provincia de Teruel de un capital social de tipo bonding propio de estructuras cerradas con relaciones redundantes entre familiares y conocidos. A la vez, no se observa una articulación fuerte con redes intermedias y exteriores que creen relaciones estratégicas entre el entramado estatal, empresarial y la sociedad civil. Ello puede suponer que, en general, la disponibilidad de capital social de tipo bridging sea limitada. Hay que recordar que el capital social de tipo bonding hace referencia a las relaciones entre grupos relativamente homogéneos, y que el capital social de tipo bridging se refiere a relaciones más distantes, menos estrechas y más diversas (Putnam, 2000); y que mientras las primeras son importantes para "ir funcionando" en el día a día, las segundas lo son para avanzar en el desarrollo socioeconómico. Por otra parte, dado que, como seńalan Marcuello et al. (2010), la realidad social es compleja y heterogénea, la realidad asociativa no responde estrictamente a los modelos típicos diseñados en teoría. Así, también surge en la provincia de Teruel otro tipo de organizaciones denominadas de forma genérica "plataformas", que sirven para articular la fuerza y los intereses tanto de individuos como de colectividades. El sentido de tales plataformas o entidades de segundo (o tercer) nivel radica, en todo caso, en ese intento de conjugar y aunar fuerzas e intereses dispersos que pueden generar capital social de tipo linking, es decir, acceder a niveles jerárquicos superiores para hacer que sus demandas sean, al menos, tenidas en cuenta y escuchadas. En este sentido, resulta recomendable la activación de las diferentes federaciones y plataformas existentes en la provincia de Teruel para movilizar a la sociedad civil.

El análisis realizado permite apreciar un desarrollo del capital social a dos velocidades muy desiguales en las comarcas que conforman la provincia de Teruel. Unas zonas entran en el bucle del despoblamiento/envejecimiento y pérdida de interacción con niveles exteriores de organizaciones sociales y empresariales, mientras que otras parecen recuperarse entrando en una dinámica de rejuvenecimiento y crecimiento del tejido social. Estos resultados indican que en la provincia de Teruel se debe realizar un esfuerzo importante por revertir esa debilidad del capital social, para lo cual hace falta un mayor empeño en coordinar acciones para el desarrollo entre las diversas Administraciones Locales. Para lograrlo se debe contar también con las Comarcas, la Administración Autonómica y otras entidades que operan en el territorio con experiencia probada en desarrollo rural, como los GAL, que agrupan a los agentes sociales de sus respectivos territorios; también con la activación de las organizaciones de segundo nivel, a fin de que se establezcan vínculos débiles entre 
las diferentes comarcas, con el resto de Aragón y con el resto de España. Como se ha señalado ya, los vínculos débiles tienen mayor importancia que las redes fuertes y cerradas cuando se trata de permitir el acceso a información y conocimiento más novedosos, ambos aspectos fundamentales para iniciar una senda de desarrollo. La sociedad turolense, junto a sus instituciones, no puede dejar de reivindicar una política de ordenación del territorio más racional y coherente, que impulse realmente un mayor equilibrio territorial y un desarrollo económico y social basado en criterios de sostenibilidad.

Un trabajo académico futuro podría centrarse en un mayor análisis de las redes establecidas por las empresas, Universidad, administración pública y tercer sector, que ayude a propiciar su consolidación o a impulsar su formación en aquellos ámbitos en los que resulte necesario. También un estudio de interés sería analizar la actuación de los GAL de la provincia de Teruel y su contribución al fortalecimiento de la sociedad civil local rural, al establecimiento de vínculos con otros agentes y a profundizar en la forma en que estos contribuyen a diversificar la economía rural y a dinamizar sus respectivos territorios de actuación.

\section{Referencias bibliográficas}

A+M (2011). Barómetro de Opinión de Aragón. Primavera de 2011. Zaragoza: Gobierno de Aragón. En http://www.aragonhoy.net/index.php/mod.noticias/mem.detalle/ id. 103299

Baquero, M. (2003). Reinventando a sociedade na América Latina. Cultura política, gênero, exclusão e capital social. Porto Alegre: Editora de Universidade, Universidade Federal de Rio Grande do Sul.

Bourdieu, P. (1980). Le capital social. Notes provisoires. Actes de la Recherche en Sciences Sociales, 3, 2-3. En http://goo.gl/Vq0yKT

Bourdieu, P. (1985). The forms of capital. En J. G. Richardson (Ed.), Handbook of Theory and Research for the Sociology of Education (pp. 241-258). Nueva York: Greenwood.

Brehm, J. \& Rahn, W. (1997). Individual-level evidence for the causes and consequences of social capital. American Journal of Political Science, 41(3), 999-1023.

Bryden, J. M. (1998). Development strategies for remote rural regions: What do we know so far? Comunicación presentada en la conferencia internacional de la OCDE: Remote rural Areas. Developing through Natural and Cultural Assets. Abarracín, España, noviembre 5-6, 1998.

Buciega, A. (2010). Capital social y LEADER: los recursos generados entre 1996y 2008. Documentos de trabajo 2010-15, DT, 33. Valencia: Instituto Interuniversitario de Desarrollo Local, Valencia. En http://www.ceddar.org/content/files/articulof_330_01_DT201015.pdf

Burillo, F. (2014). Territorio despoblado. Heraldo de Aragón, 2 de julio de 2014, p. 21.

Burillo, M. P., Burillo, F. \& Ruiz, E. (2013). Serrania Celtibérica (España). Un proyecto de desarrollo rural para la Laponia del Mediterráneo. Teruel: Instituto Celtiberia de Investigación y Desarrollo Rural, Campus de Teruel.

Burt, R. S. (2005). Brokerage and closure. Oxford: Oxford University Press. 
Camagni, R. (2003). Incertidumbre, capital social y desarrollo local: enseñanzas para una gobernabilidad sostenible del territorio. Investigaciones Regionales, 2, 31-57. En dialnet. unirioja.es/descarga/articulo/2123586.pdf

Camagni, R. (2008). Regional competitiveness: towards a concept of territorial. En R. Capello, R. Camagni, B. Chizzolini \& U. Fratesi (Eds.), Modelling regional scenarios for the enlarged Europe: European competitiveness and global strategies (pp. 33-47). Berlín: Springer.

Centro de Investigaciones Sociológicas (CIs). (2010). Estudio no 2844. Madrid: CIs.

Coleman, J. (1988). Social capital and the creation of human capital. American Journal of Sociology, 94, S95-S120. En http://goo.gl/Mif8F2

Coleman, J. (1990). Foundation of Social Theory. Cambridge: Harvard University Press.

Comisión Económica para América Latina y el Caribe (CEPAL). (1992). El desarrollo sustentable.

Transformación productiva, equidad y medio ambiente. Santiago de Chile: CEPAL-ONU. En http://goo.gl/Mif8F2

Dasgupta, P. (1999). Economic progress and the idea of social capital. En P. Dasgupta \& I. Serageldin (Eds.), Social capital: A multifaceted perspective (325-424). Washington, D.C.: The World Bank.

Dijkstra, L. \& Poelman, H. (2011). Regional typologies: a compilation. European Unión Regional Policy, 1. Working Papers. A series of short papers on regional research and indicators produced by the Directorate-General for Regional Policy. En http://goo.gl/SblQaI

Dirección General de Atención a la dependencia. (2010). La dependencia en Aragón, periodo de 2007 a 2009. Gobierno de Aragón, Departamento de Servicios sociales y Familia. En http://goo.gl/yv5 $\mathrm{YbH}$

Durston, J. (1999). Construyendo capital social comunitario. Revista de la cepal, 69, 103-118. En http://www.cepal.org/publicaciones/SecretariaEjecutiva/7/lcg2067/durstonesp.pdf

Durston, J. (2000). ¿Qué es capital social comunitario? Serie Políticas Sociales, 38 (LC/L. 1400-P). Santiago de Chile: División de Desarrollo Social: Cepal. En http://www. cepal.org/publicaciones/xml/5/4885/lcl1400.pdf

Falk, I. \& Kilpatrick, S. (2000). What is social capital? A study of interaction in a rural community. Sociologia Ruralis, 40, 87-110. doi: 10.1111/1467-9523.00133

Fukuyama, F. (1995). Trust: The social virtues and the creation of prosperity. Nueva York: Free Press.

Gambetta, D. (1988). Trust: making and breaking of cooperative relationships. Oxford: Basil Blackwell.

Garofoli, G. (1992). Endogenous development and Southern Europe. Aldershot: Avebury.

Gobierno de Aragón, Departamento de Agricultura, Ganadería y Medio Ambiente (2014). Spain - Rural Development Programme (Regional) - Aragón. En http://goo.gl/hZBJWy

Gómez-Quintero, J. D. \& Sanz-Gracia, F. (2013). Situación socioeconómica de Teruel: presente y futuro. Edición digital. Consejo Económico y Social de Aragón. En http://goo.gl/cSQqtE Granovetter, M. (1973). The strength of weak ties. American Journal of Sociology, 78, 1360-1380. Granovetter, M. (1985). Economic action and social structure: the problem of embeddedness. American Journal of Sociology, 91(3), 481-510. En http://goo.gl/ZQW4HX

Growiec, K. \& Growiec, J. (2014). The impact of bridging and bonding social capital on individual earnings: Evidence for an inverted U. NBP Working Paper, 175. Economic Institute Warsaw: Narodowy Bank Polski.

Hall, P. (1999). Social capital in Britain. British Journal of Political Science, 29(3), 417-461. 
Herreros, F. \& De Francisco, A. (2001). Introducción: el capital social como programa de investigación. Zona Abierta 94/95, 1-46.

Hooghe, M. (2003). Participation in voluntary associations and value indicators: The effects of current and previous participation experiences. Nonprofit and Voluntary Sector Quarterly, 32, 47-69.

Instituto Aragonés de Estadística (IAEST). (2011). Datos básicos de Aragón. Elecciones. Última actualización febrero de 2012. Cifras de elecciones municipales año 2011, con datos del Ministerio del Interior. En http://goo.gl/n3Dnso

Instituto Aragonés de Estadística (IAEST)/Gobierno de Aragón. (2000). Estadística del sector no lucrativo en Aragón. Año 2000. En http://goo.gl/RCyTzB

Instituto Aragonés de Estadística (IAEST)/Gobierno de Aragón. (2011). Datos Básicos de Aragón. www.es/iaest. Última actualización, abril de 2013. En http://goo.gl/jj8WTp

Instituto Nacional de Estadística (INE), España. (2008). Encuesta sobre Discapacidades y Autonomía personal y situaciones de Dependencia 2008. Madrid: INE. En http://goo. $\mathrm{gl} / 7 \mathrm{Qj} 5 \mathrm{e}$

Leonard, R. \& Onyx, J. (2003). Networking through loose and strong ties: An Australian qualitative study. Voluntas: International Journal of Voluntary and Nonprofit Organizations, 14(2), 189-203.

Lewicki, R. J., Mcallister, D. J. \& Bies, R. J. (1998). Trust and distrust: New relationships and realities. Academy of Management Review, 23, 438-458. doi:10.5465/AMR.1998.926620

López, A., Ansó, J. L., Minguijón, J., Nocetti, C. \& Ulldemolíns, E. (2006). Las Comarcas: Una nueva organización territorial de Aragón. Estado de las funciones y servicios transferidos a la administración comarcal 2002-2005. Zaragoza: Grupo Sociológico de Investigación Científica. Universidad de Zaragoza. En http://goo.gl/Ym6NVX

Maillat, D. (1995). Les milieux innovateurs. Sciences Humaines, 8, 41-42.

Malecki, E. J. (2011). Regional social capital: Why it matters. Regional Studies, 46(8), 1023 1039. doi: 10.1080/00343404.2011.607806

Marcuello, C., Marcuello, Ch., Bellostas, A. \& Moneva, M. (2007). Capital social y organizaciones no lucrativas en España. El caso de las ongd. Bilbao: Fundación вBVA.

Marcuello, C., Díaz, M., Bellostas, A., Filgueira, M., García, A., Martínez, M., Marcuello, Ch., Saz, I. \& Serrano, C. (2010). Plataformas aragonesas: Análisis desde el enfoque de la gobernanza y los procesos de participación. Informe final. Grupo de Estudios Sociales y Económicos del Tercer Sector (geses), Universidad de Zaragoza. En http://goo.gl/qZokUu

Monfort, Ph. (2009). Territories with specific geographical features. European Union Regional Policy, 2. Working Papers. A series of short papers on regional research and indicators produced by the Directorate-General for Regional Policy. En: http://ec.europa.eu/ regional_policy/sources/docgener/work/2009_02_geographical.pdf

Montero, J. R., Calvo, K. \& Martínez, Á. (2008). El voto religioso en Espańa y Portugal. Revista Internacional de Sociología, 66(51), 19-54. doi: 10.3989/ris.2008.i51.108

Ostrom, E. (1994). Constituting social capital and collective action. Journal of Theoretical Politics, 6, 527-562. doi: 10.1177/0951692894006004006

Ostrom, E. (1999). Social capital: A fad or a fundamental concept. En P. Dasgupta y I. Serageldin (Eds.), Social Capital: A Multifaceted Perspective, Washington, D.c.: World Bank. 
Pereira, D., Fernández, F., Ocón, B. \& Márquez, O. (2004). Las zonas rurales en España. Un diagnóstico desde la perspectiva de las desigualdades territoriales y los cambios sociales y económicos. Madrid: Fundación Fomento de Estudios Sociales y de Sociología Aplicada (fOESSA)/Cáritas Española Editores.

Portes, A. (2004). El futuro de América Latina. Neoliberalismo, clases sociales y transnacionalismo. Bogotá: Instituto Latinoamericano para una Sociedad y Derechos Alternativos (ILSA).

Putnam, R. (1993). Making democracy work: Civic traditions in modern Italy. Princeton, NJ: Princeton University Press.

Putnam, R. (1995). Bowling alone: America's declining social capital. Journal of Democracy, 6(1), 65-78. En http://goo.gl/aQ0C0o

Putnam, R. (2000). Bowling alone: The collapse and revival of American community. Nueva York: Simon and Schuster.

Querol, J. V. (2006). Acciones de desarrollo rural en la provincia de Teruel iniciativas comunitarias e iniciativas locales. En L. M. Frutos \& E. Ruiz (Coord.), Estrategias Territoriales de desarrollo rural (pp.125-144). Zaragoza: Institución "Fernando el Católico", Excma. Diputación de Zaragoza.

Sanders, J. M. \& Nee, V. (1996). Immigrant self-employment: The family as social capital and the value of human capital. American Sociological Review, 61(2), 231-249.

Saz Gil, M. I. (2007). El capital social en las Organizaciones No Lucrativas. Implicaciones en la gestión: una aproximación a través del estudio de casos. Tesis doctoral. Universidad de Valencia: Servei de Publicacions.

Sforzi, F. (2002). Los distritos industriales ante el reto de la globalización. Geographicalia, 41, 5- 18. En http://dialnet.unirioja.es/servlet/articulo?codigo $=257335$

Shaw, R. (1997). Trust in the balance. San Francisco, Ca: Jossey-Bass Publishers.

Skocpol, T. (1997). The Tocqueville problem. Civic engagement in American democracy. Social Science History, 21(4), 456-479. En https://www2.southeastern.edu/Academics/ Faculty/jbell/skocpol.pdf

Sobel, J. (2002). Can we trust social capital? Journal of Economic Literature, Vol. XL, 139-154.

Stolle, D., \& Rochon, T. (1998). Are all associations alike? Member diversity, associational type and the creation of social capital. American Behavioral Scientists, 42(1), 47-61. doi: $10.1177 / 0002764298042001005$

Sweeney, G. (2001). Social capital: the core factor in economic resurgence. En G. Sweeney (Ed.), Innovation, economic progress and the quality of life (pp. 144-163). Cheltenham: Edward Elgar.

Torcal, M. \& Montero, J. (2000). La formación y consecuencias del capital social en España. Revista Española de Ciencia Política, 1(2), 79-121. En http://www.recp.es/index.php/ recp/article/view/305

Triglia, C. (2001). Social capital and local development. European Journal of Social Theory, 4(4), 427-442. doi: 10.1177/13684310122225244

Uphoff, N. (2000). Understanding social capital: Learning from the analysis and experience of participation. En P. Dasgupta \& I. Serageldind (Eds.), Social capital: A multifaceted perspective (pp. 215-249). Washington, D.c.: Banco Mundial.

Van Deth, J. W. (2006). Democracy and involvement. The benevolent aspects of social participation. En M. Torcal, M. \& J. M. Montero (Eds.), Political disaffection in contemporary democracies (pp. 101-129). Londres: Routledge. 
Warner, M. (2001). Building social capital: the role of local government. Journal of SocioEconomics, 30(2), 187-192. En http://goo.gl/9Esvhp

Westlund, H. (2006). Social capital in the knowledge economy: Theory and empirics. Berlín: Springer.

Woolcock, M. (1998). Social capital and economic development: toward a theoretical synthesis and policy framework. Theory and Society, 27, 151-208. doi 10.1023/A:1006884930135

Woolcock, M. (2001). The place of social capital in understanding social and economic outcomes. En Organisation for Economic Co-operation and Development (OECD), The contribution of human and social capital to sustained economic growth and well-being (pp. 65-88). International Symposium Report, September.

Woolcock, M. \& Narayan, D. (2000). Social capital: Implications for development theory, research and policy. The World Bank Research Observer, 15(2), 225-250. doi: 10.1093/ wbro/15.2.225

Yamagishi, T. \& Yamagishi, M. (1994). Trust and commitment in the United States and Japan. Motivation and Emotion, 18(2), 129-166. En http://goo.gl/yU8ZwX

Zucker, L. G. (1986). Production of trust: Institutional sources of economic structure, 18401920. Research in Organizational Behavior, 8, 53-111. 
